\title{
COMET muon conversion experiment in J-PARC
}

\section{MyeongJae Lee*i}

Institute for Basic Science, Daejeon, Korea

E-mail: myeongjaelee@ibs.re.kr

COMET is an experiment at J-PARC, Japan, which will search for neutrinoless conversion of muons into electrons in the field of a nucleus $\left(\mu^{-}+N \rightarrow e^{-}+N\right)$; a lepton flavor violating process. The experimental sensitivity goal for this process is of order $10^{15}$ for Phase-I and $10^{17}$ for Phase-II experiment, which is a factor of 100 to 10,000 improvements correspondingly over existing limits. Recent progress in facility and detector development are presented, along with COMET Phase-I experimental schedule.

The 19th International Workshop on Neutrinos from Accelerators-NUFACT2017

25-30 September, 2017

Uppsala University, Uppsala, Sweden

*Speaker.

${ }^{\dagger}$ On behalf of COMET collaboration 


\section{Introduction}

The COMET (COherent Muon to Electron Transition, J-PARC E21) experiment [1] aims to search for neutrinoless conversion of muon into electron in a muonic atom $\left(\mu^{-}+N \rightarrow e^{-}+N\right.$; $\mu-e$ conversion). This is one of important charged lepton flavor violating (cLFV) processes of muon, along with $\mu^{+} \rightarrow e^{+} \gamma$ and $\mu^{+} \rightarrow e^{+} e^{-} e^{+}$processes. A diagram of Standard Model process of $\mu-e$ conversion is shown in the left figure of Fig. 1, where the process is suppressed down to $O\left(10^{-54}\right)$ due to heavy W-boson mass and tiny probability of neutrino oscillation. In comparison, the right figure of Fig. 1 represents the possible $\mu-e$ conversion in Supersymmetry (SUSY) theory, which might be enhanced depending on the masses and interactions of mediating SUSY particles. Therefore, the observation of $\mu-e$ conversion process is a direct evidence of the theories beyond Standard Model.

COMET experiment is locating at Japan Proton Accelerator Research Complex (J-PARC) in Tokai, Japan. The $8 \mathrm{GeV}$ bunched proton beam from the main ring (MR) of J-PARC will be supplied to the Nuclear Physics Experiment hall (NP hall) where COMET facility and detectors are locating. The COMET experiment will be carried out by two stages. The first stage (Phase-I) will utilize half length transport solenoid and a drift chamber detector for measuring $\mu-e$ conversion signal down to $O\left(10^{-15}\right)$. In the second stage (Phase-II), full length C-shape transport solenoid, spectrometer solenoid, and straw tube detectors will be used to achieve $O\left(10^{-17}\right)$ level of $\mu-e$ conversion measurement. These target sensitivities are 100 or 10,000 times better than the current experimental world limit given by SINDRUM-II experiment [2]. In this proceeding, we present the status and plan of COMET Phase-I experiment, where the detectors and facilities are described in Sec. 2, and its physics reach, in Sec. 3.

\section{COMET detector and facility}

\subsection{Accelerator and Proton beam line}

COMET experiment will produce muon beam from the decay of pions generated at the proton target through the interaction with $8 \mathrm{GeV}$ protons. These protons are extracted from the MR of J-PARC to the NP hall ("Slow Extraction"), which get filled from the $3 \mathrm{GeV}$ Synchrotron. Table 1 summaries the specification of proton beam. The most important specification of proton beam in $\mu-e$ conversion experiment is the near-complete extinction of proton particles between proton
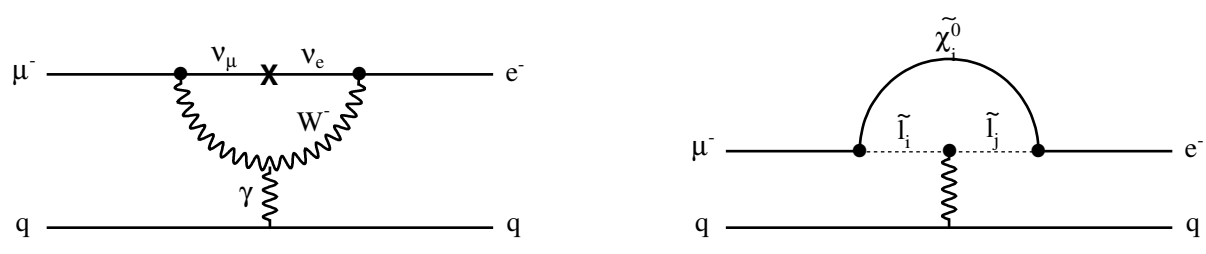

Figure 1: The diagrams of Standard Model process (left figure) and Supersymmetry theory (right figure) of $\mu^{-}+N \rightarrow e^{-}+N$. 
pulses. As the muons captured in muon stopping target materials decay with finite life time in the order of a few $100 \mathrm{~ns}$, a pre-defined measurement time window are applied in $\mu-e$ conversion measurement to avoid high detector hit rate and background hits coming from the proton pulse. The protons between pulses coinciding with measurement time window affect directly to the $\mu-e$ conversion measurement, therefore, high extinction of protons between pulses are preferred. The requirement of this extinction factor in COMET experiment is $O\left(10^{-9}\right)$, where extinction measurement at the proton extraction line to long-baseline neutrino experiments ("Fast extraction") have achieved $O\left(10^{-11}\right)$.

Table 1: Proton beam specifications in COMET Phase-I experiment.

\begin{tabular}{cc|cc}
\hline \hline Beam power & $3.2 \mathrm{~kW}$ & Energy & $8 \mathrm{GeV}$ \\
Average current & $0.4 \mu \mathrm{A}$ & Beam emittance & $10 \pi \mathrm{mm} \cdot \mathrm{mrad}$ \\
Proton per bunch & $<10^{10}$ & Extinction & $10^{-9}$ \\
Bunch spacing & $1 \sim 2 \mu \mathrm{sec}$ & Bunch length & $100 \mathrm{~ns}$ \\
\hline \hline
\end{tabular}

\subsection{Pion and Muon beam line}

The proton target, pion capture solenoid, and muon transport solenoid systems are shown in Fig. 2. The Graphite proton target system is designed so that it can be replaced in PhaseII experiment, where Tungsten target will be employed. The target is surrounded by pion/muon capture solenoid with gradient magnetic field, of which the field strength at the most upstream part and near the entrance to transport solenoid are $5 \mathrm{~T}$ and $3 \mathrm{~T}$, respectively. This enables collection of pions with backward direction, increasing muon yield per proton.

The pions generated at the proton target passes the muon transport solenoid system. In COMET Phase-II, a complete C-shaped transport solenoid with $180^{\circ}$ will be installed, however, the half length $90^{\circ}$ solenoid will be used in the Phase-I experiment, as an staged approach of solenoid construction. In addition to the $3 \mathrm{~T}$ solenoidal field, vertical dipole field is added. As the helix trajectories of pions and muons drift to the perpendicular direction to beam line plane, those trajectories should be restored to the baseline to enter to detector solenoid in position. This is achieved by applying around 500 Gauss dipole field. This muon transport solenoid system is installed in COMET hall in 2016.

\subsection{The COMET Phase-I detectors}

Two separate detector systems are under development for COMET Phase-I experiment. For measuring $\mu-e$ conversion, the CyDET detector system will be used. After the experiment, a special background measurement program is planned in order for direct estimation of beam-related background and application of the estimation to Phase-II experiment. The detector for background measurement program is called StrECAL. StrECAL is composed of straw-tube detector planes and LYSO (Lutetium-Yttrium Oxyorthosilicate) calorimeter array, for measuring particle momentum and energy, respectively. It basically resembles the Phase-II detector system. Several times of integrated beam test with the prototypes of straw-tube detector, calorimeter, and trigger and readout system were performed successfully, during 2016 and 2017. 


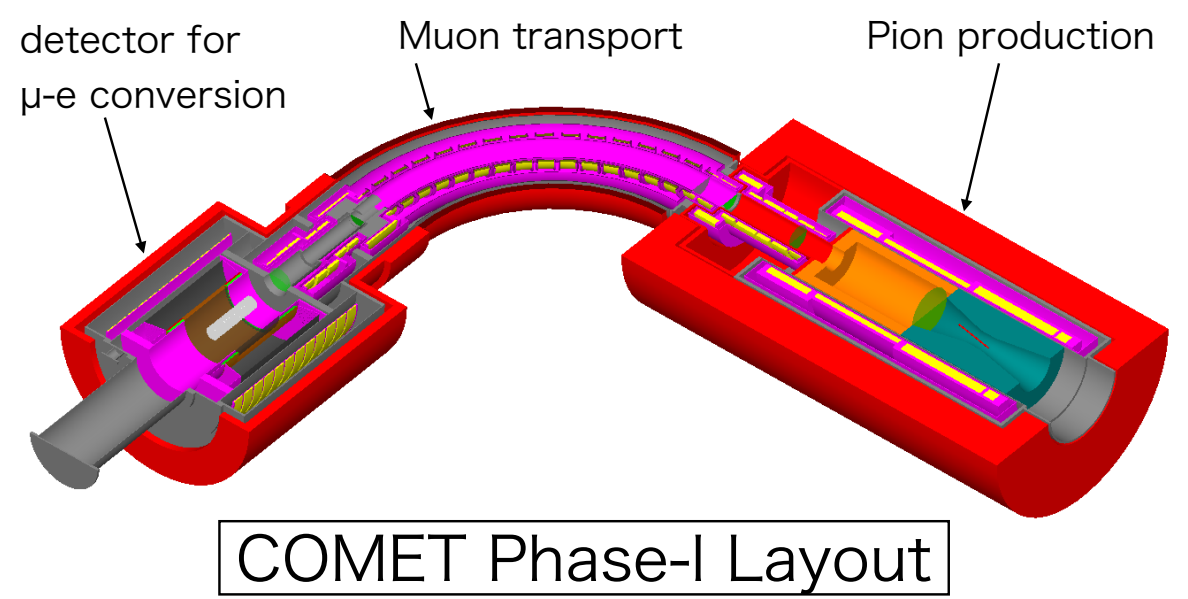

Figure 2: The layout of detectors and beamlines of COMET Phase-I experiment. The pion capture solenoid system with proton target in the center (right part), the muon transport solenoid system (middle part), and the CyDET detector system with muon stopping target in the center (left part) are shown.

The CyDET (Fig. 3) is composed of Cylindrical Drift Chamber (CDC) and trigger hodoscope array. At the center of CDC is the muon stopping targets of Aluminum disks, where the muons are captured and $\mu-e$ conversion is occurring. Around 5000 wires of CDC are structured into 18 layers, with Helium based gas filled. Two trigger hodoscope arrays surround both inner edges of CDC, where each array is composed of 48 sets of scintillator and Cerenkov detector pairs. When $\mu-e$ conversion occurs, the helical trajectory inside $1 \mathrm{~T}$ solenoidal field generates CDC hits and subsequently hodoscope hits. The CDC is constructed successfully in 2016, and the spatial resolution better than $200 \mu \mathrm{m}$ is measured during cosmic ray tests.

\section{Physics reach of COMET Phase-I experiment}

The signal of $\mu-e$ conversion is single monoenergetic electron with an energy of:

$$
E_{\mu e}=m_{\mu}-B_{\mu}-E_{\text {recoil }}=104.97 \mathrm{MeV} \text { (Aluminum target case) }
$$

where $m_{\mu}$ is mass of muon, $B_{\mu}$ is binding energy of muonic atom is $1 \mathrm{~s}$ state, and $E_{\text {recoil }}$ is energy loss of emitting electron due to the recoil of nuclei. The momentum of signal (or background) electron will be measured by CDC of CyDET in the case of COMET Phase-I, or by straw detector of StrECAL in the case of COMET Phase-II. The electron track and its momentum can be reconstructed by applying hit selection, track finding, and track fitting algorithms. The signal acceptance of Phase-I experiment is estimated using GEANT-4 based simulation framework ("ICEDUST"), and Tab. 2 shows the result. The major inefficiencies are coming from the geometry of CDC which is not $4 \pi$ detector, and the short measurement time window due to beam cycle. The overall signal acceptance is around $4.1 \%$.

For the background events of COMET experiment, they can be categorized into physics backgrounds and beam-related backgrounds. The physics backgrounds are consequence of muonic 


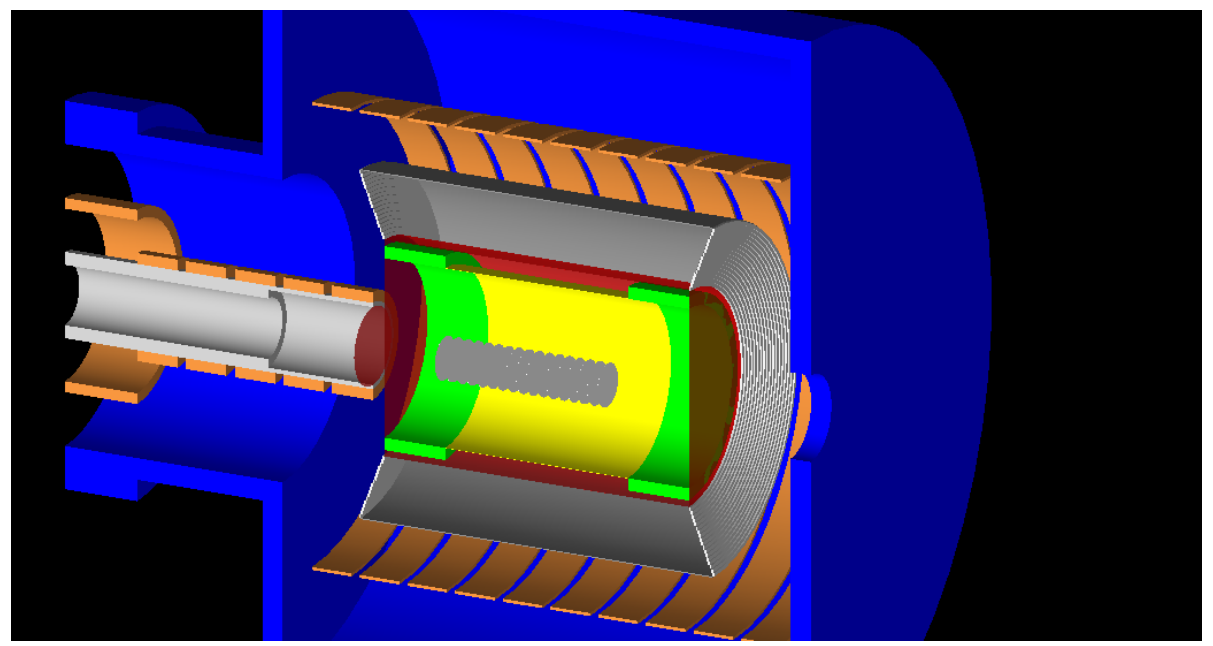

Figure 3: The layout of CyDET detector system. The gray cylindrical parts are CDC, two green rings at CDC inner edges are trigger hodoscope, gray disks at the center of CDC are muon stopping target, and orange rings around $\mathrm{CDC}$ are solenoid coils.

Table 2: Breakdown of the $\mu-e$ conversion signal acceptances.

\begin{tabular}{llc}
\hline \hline Event selection & Value & Comments \\
\hline Online event selection efficiency & 0.9 & \\
DAQ efficiency & 0.9 & \\
Track finding efficiency & 0.99 & \\
Geometrical acceptance + Track quality cuts & 0.18 & \\
Momentum window & 0.93 & $103.6 \mathrm{MeV} / c<P_{e}<106.0 \mathrm{MeV} / c$ \\
Timing window & 0.3 & $700 \mathrm{~ns}<t<1170 \mathrm{~ns}$ \\
\hline Total & 0.041 & \\
\hline \hline
\end{tabular}

atom. The muons in muonic atoms can decay without interaction with nuclei, into one electron and two neutrinos, which is called Decay-in-Orbit (DIO) background. The energy distribution of DIO electron peaks at $52 \mathrm{MeV}$, however extends up to $105 \mathrm{MeV}$ due to the recoil of nuclei. This high energy tail of DIO is non-reducible physics background source. The other possibility of muon in muonic atom is its capture to nuclei, meaning that it decays with Weak interactions with neutrons in nuclei leaving them excited states and decaying with secondary particle emission. Specially the secondary $\gamma$ may have enough energy to create $e^{-}-e^{+}$pair, and those electrons with similar energy with $\mu-e$ conversion signal will be background. This is called Radiative Muon Capture (RMC). The beam-related background are effects of non-muon particles in the beam and their decay products, or muon decay effects during transportation to the muon stopping target. Specially, the pions in the beam can be captured in the stopping target, decays with $\gamma$ radiation which may create $e^{-}-e^{+}$pair. This is called Radiative Pion Capture (RPC).

While DIO is non-reducible, COMET Phase-I experiment is subject to rather higher RPC background, due to short length of pion transportation. All other backgrounds are distributed flat in 
the $\mu-e$ conversion signal region, when DIO shows rapidly dropping spectrum nearly up to 105 $\mathrm{MeV}$. Therefore, to reduce the effects of DIO, it is critical to have enough momentum resolution of electron measurement. Table 3 summarizes estimated background events from GEANT-4 based simulation. The total number of background events estimated in the $\mu-e$ conversion signal region is 0.032 .

Table 3: Summary of the estimated background events for a single-event sensitivity of $3 \times 10^{-15}$ in COMET Phase-I with a proton extinction factor of $3 \times 10^{-11}$.

\begin{tabular}{llr}
\hline \hline Type & Background & Estimated events \\
\hline Physics & Muon decay in orbit & 0.01 \\
& Radiative muon capture & 0.0019 \\
& Neutron emission after muon capture & $<0.001$ \\
& Charged particle emission after muon capture & $<0.001$ \\
\hline Prompt Beam & Radiative pion capture & 0.0028 \\
& Neutrons & $\sim 10^{-9}$ \\
& All others including Muon/pion decay in flight & $\leq 0.0038$ \\
\hline Delayed Beam & Beam electrons & $\sim 0$ \\
& Muon decay in flight & $\sim 0$ \\
& Pion decay in flight & $\sim 0$ \\
& Radiative pion capture & $\sim 0$ \\
& Anti-proton induced backgrounds & 0.0012 \\
\hline Others & Cosmic rays & $<0.01$ \\
\hline Total & & 0.032 \\
\hline \hline
\end{tabular}

Figure 4 shows the estimated DIO background spectrum and $\mu-e$ conversion signal spectrum assuming $3 \times 10^{-15}$ conversion rate. Other backgrounds are not shown in this plot, as their contributions are negligible. It is also evident that signal and DIO background separation will be clear due to enough momentum resolution of CDC.

Based on these acceptance and background estimations, the Single Event Sensitivity (SES) can be obtained by:

$$
B\left(\mu^{-}+\mathrm{Al} \rightarrow e^{-}+\mathrm{Al}\right)=\frac{1}{N_{p} \cdot R_{\mu p} \cdot f_{\text {cap }} \cdot f_{\text {gnd }} \cdot A_{\mu-e}}
$$

where $N_{p}=3.2 \times 10^{19}$ is the total number of protons, $R_{\mu p}=4.7 \times 10^{-4}$ is muon yield per proton obtained from simulation, $f_{\text {cap }}=0.61$ is the fraction of captured muon to the total muons on target, $f_{\text {gnd }}=0.9$ is the fraction of muon conversion to the ground state, and $A_{\mu-e}=0.041$ is the signal acceptance. The estimated SES is

$$
\begin{array}{rlrl}
B\left(\mu^{-}+\mathrm{Al} \rightarrow e^{-}+\mathrm{Al}\right) & =3 \times 10^{-15} & & (\text { as SES }) \text { or } \\
& <7 \times 10^{-15} \quad(\text { as } 90 \% \text { C.L. upper limit }) .
\end{array}
$$




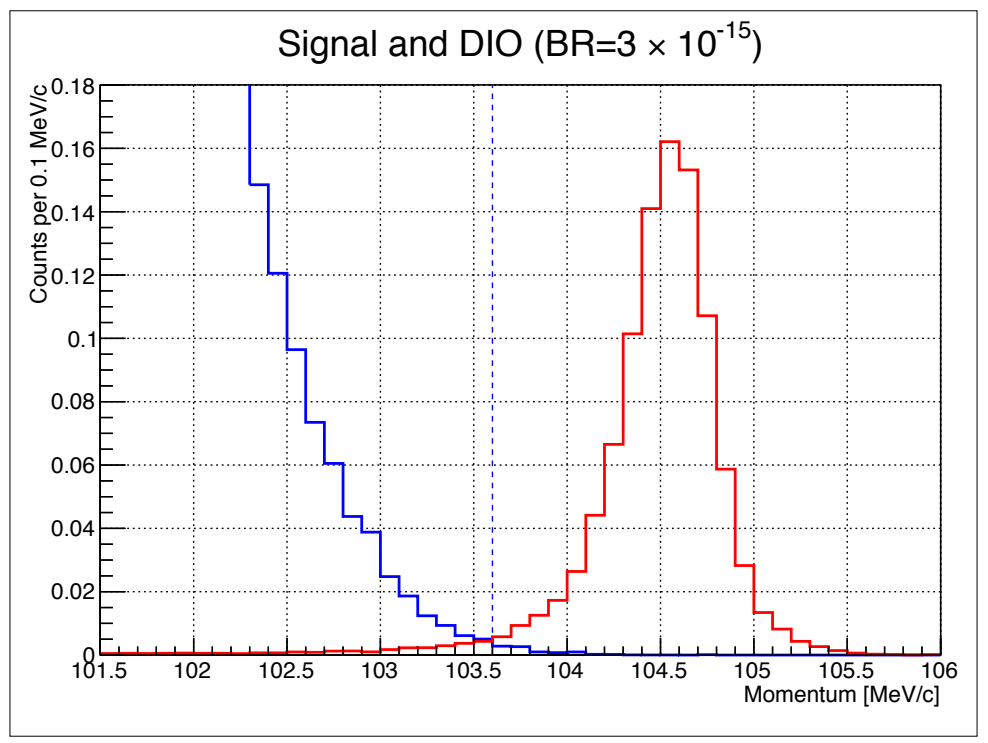

Figure 4: The momentum distributions for the reconstructed $\mu-e$ conversion signals (right solid red curve with peaking distribution) and reconstructed DIO events (left solid blue curve with decreasing distribution). The vertical scale is normalized such that the integral of the signal curve is equal to one event. This assumes a branching ratio of $B(\mu N \rightarrow e N)=3.1 \times 10^{-15}$.

\section{Summary and Prospects}

The COMET experiment is a search for $\mu-e$ conversion, which is one of important cLFV search. It utilizes $8 \mathrm{GeV}$ proton beam from J-PARC to produce muons, which will be captured in Aluminum target for muon conversion. The muon conversion signal will be measured using Cylindrical drift chamber detector in the Phase-I experiment. The estimated single event sensitivity is $3 \times 10^{-15}$ which is 100 times improvement than current world limit. The detector and facility construction is on schedule. Some of important parts such as muon transport solenoid and drift chamber are ready and operational. Other components will be ready until 2019, and half year data taking will follow. The COMET Phase-II development is also in progress, for example, the prototype detector test is completed. The data taking of Phase-II experiment will be performed after 2021 during one year, where it will be possible to reach $O\left(10^{-17}\right)$ sensitivity.

\section{Acknowledgements}

The COMET collaboration acknowledge supports from JSPS, Japan; NSFC, China; IHEP, China; IN2P3-CNRS, France; IN2P3-CC, France; SRNSF, Georgia; IBS, Korea; RFBR, Russia; STFC, United Kingdom; and Royal Society, United Kingdom.

\section{References}

[1] R. Abramishvili et al., COMET Phase-1 Technical Design Report, KEK Report 2015-1 (2015).

[2] W. Bertl et al., A search for $\mu$ - e conversion in muonic gold, EPJ C47 337 (2006). 